\title{
Dehydrocorydaline promotes myogenic differentiation via p38 MAPK activation
}

\author{
MIRAN YOO ${ }^{1}$, SANG-JIN LEE ${ }^{1}$, YONG KEE KIM ${ }^{1}$, DONG-WAN SEO ${ }^{2}$, \\ NAM-IN BAEK ${ }^{3}$, JAE-HA RYU ${ }^{1}$, JONG-SUN KANG ${ }^{4}$ and GYU-UN BAE ${ }^{1}$ \\ ${ }^{1}$ Research Center for Cell Fate Control, College of Pharmacy, Sookmyung Women's University, Seoul 140-742; \\ ${ }^{2}$ Department of Biochemistry, College of Pharmacy, Dankook University, Cheonan, Chungcheongnam 330-714; \\ ${ }^{3}$ Department of Oriental Medicine, The Graduate School of Biotechnology, Institute of Life Sciences \& Resources, \\ Kyung Hee University, Yongin, Gyeonggi 446-701; ${ }^{4}$ Department of Molecular Cell Biology, \\ Sungkyunkwan University School of Medicine, Samsung Biomedical Research Institute, \\ Suwon, Gyeonggi 440-746, Republic of Korea
}

Received July 11, 2015; Accepted August 5, 2016

DOI: $10.3892 / \mathrm{mmr} .2016 .5653$

\begin{abstract}
Muscle regeneration is a coordinated process that involves proliferation and differentiation of muscle progenitor cells. Activation of $\mathrm{MyoD}$ is a key event in myogenic differentiation, which is regulated by $\mathrm{p} 38$ mitogen-activated protein kinases (MAPK). In a screen of natural compounds for the enhancement of MyoD activity, dehydrocorydaline (DHC) from the Corydalis tuber was identified. Treatment of $\mathrm{C} 2 \mathrm{C} 12$ myoblasts with DHC increased the expression levels of muscle-specific proteins, including $\mathrm{MyoD}$, myogenin and myosin heavy chain. In addition, $\mathrm{C} 2 \mathrm{C} 12$ myoblasts exhibited enhanced multinucleated myotube formation without any cytotoxicity. Treatment with DHC elevated p38 MAPK activation and the interaction of MyoD with an E protein, which is likely to result in activation of MyoD and promotion of myoblast differentiation. Furthermore, defects in differentiation-induced p38 MAPK activation and myoblast differentiation induced by depletion of the promyogenic receptor protein Cdo in $\mathrm{C} 2 \mathrm{C} 12$ myoblasts were restored by DHC treatment. In conclusion, these results indicated that DHC stimulates p38 MAPK activation, which can enhance heterodimerization of $\mathrm{MyoD}$ and $\mathrm{E}$ proteins, thus resulting in
\end{abstract}

Correspondence to: Professor Gyu-Un Bae, Research Center for Cell Fate Control, College of Pharmacy, Sookmyung Women's University, 100 Cheongpa-ro 47-gil, Seoul 140-742, Republic of Korea

E-mail: gbae@sookmyung.ac.kr

Professor Jong-Sun Kang, Department of Molecular Cell Biology, Sungkyunkwan University School of Medicine, Samsung Biomedical Research Institute, 300 Cheoncheon-Dong, Suwon, Gyeonggi 440-746, Republic of Korea

E-mail:kangj01@skku.edu

Key words: Corydalis tuber, dehydrocorydaline, myoblast differentiation, MyoD, p38 MAPK
MyoD activation and myoblast differentiation. These findings suggested that DHC may be considered a potential therapeutic compound for the improvement of muscle stem cell regenerative capacity in injured muscle.

\section{Introduction}

Loss of skeletal muscle mass, also known as atrophy, may occur in normal aging-related conditions or in chronic pathological conditions, including myopathy, denervation-associated atrophy, cachexia and obesity $(1,2)$. Skeletal muscle atrophy is associated with increased fatigability and metabolic health problems leading to a reduced quality of life, which represents a major public health burden in several countries. Therefore, great efforts have been made to identify therapeutic tools to prevent or retard muscle atrophy. Muscle regeneration is a coordinated process that involves proliferation and differentiation of muscle progenitor cells. Skeletal myoblast differentiation is a multistep process that is associated with cell cycle exit, muscle-specific gene expression, and formation of multinucleated myotubes via myoblast fusion (3). Myogenesis is well-orchestrated by the myogenic basic helix-loop-helix transcription factors, including MyoD, myogenin and myogenic factor 5 (4). Mice lacking MyoD exhibit delayed myogenesis in the limbs and branchial arches (2). The activation of MyoD is a key regulatory step for the induction of myoblast differentiation. Notably, p38 mitogen-activated protein kinases (MAPK) have a fundamental role in muscle differentiation via the activation of chromatin remodeling proteins and myogenic transcription factors, such as MyoD (5). p38 MAPK induces the heterodimerization of $\mathrm{MyoD}$ with E proteins, thus resulting in upregulation of muscle-specific genes, including myogenin and myosin heavy chain (MHC) $(6,7)$. Various promyogenic cell surface signaling pathways, such as Cdo-mediated cell adhesion signaling, activate p38 MAPK thereby inducing myoblast differentiation (8).

Corydalis tuber, which is the rhizome of Corydalis turtschaninovii, has been used to treat 
inflammatory, hemorheological and allergic diseases in Korea and China $(9,10)$. It has previously been reported that several alkaloid compounds can be isolated from Corydalis tuber $(10,11)$. Among these, dehydrocorydaline (DHC) has been demonstrated to suppress the elevated mitochondrial membrane potential in lipopolysaccharide-stimulated macrophages (12), and to inhibit proliferation of breast cancer cells by inducing apoptosis (13). However, the effects of DHC on myoblast differentiation have yet to be described.

In the present study, DHC, which is an isoquinoline alkaloid, was selected in a screening of natural phytochemicals purified from the Corydalis tuber (Papaveraceae) for the activation of MyoD-responsive reporters and induction of MHC in myoblasts. Subsequently, the effects of DHC on myoblast differentiation and the underlying regulatory mechanisms were investigated. Treatment of $\mathrm{C} 2 \mathrm{C} 12$ myoblasts with $\mathrm{DHC}$ enhanced the differentiation-linked activation of p38 MAPK and elevated the interaction of MyoD with $\mathrm{E}$ proteins, thus resulting in promotion of myoblast differentiation. In addition, DHC treatment rescued p38 MAPK activation and multinucleated myotube formation in Cdo-depleted $\mathrm{C} 2 \mathrm{C} 12$ cells. The present study is the first, to the best of our knowledge, to report that phytochemical DHC promotes MyoD-mediated myogenesis via activation of the p38 MAPK promyogenic signaling pathway.

\section{Materials and methods}

Preparation of DHC from Corydalis tuber. The Corydalis tuber was purchased from Kyungdong Herb Medicine Market (Seoul, South Korea) and was authenticated by Professor Dae-Keun Kim (Woosuk University, Jeonju, South Korea). A voucher specimen (KHU070123) was reserved at the Laboratory of Natural Products Chemistry (Kyung Hee University, Yongin, South Korea). The powdered tuber $(5 \mathrm{~kg})$ was extracted with $80 \%$ aqueous $\mathrm{MeOH}(5.0 \mathrm{Lx} 2)$ at room temperature to give a dark brownish extract $(347 \mathrm{~g})$. The methanol extract was then poured into acidic water $(\mathrm{pH} 2.5 ; 2.0 \mathrm{~L})$ and was washed twice with EtOAc $(2.0 \mathrm{Lx} 2)$. The $\mathrm{pH}$ of the aqueous layer was increased to $\mathrm{pH} 12.0$ using $20 \% \mathrm{NaOH}$ solution, and was then extracted with EtOAc (2.0 Lx2) and $n-\mathrm{BuOH}$ (1.5 Lx2) successively. The $n$-BuOH soluble layer (CTB; $58 \mathrm{~g}$ ) was subjected to $\mathrm{SiO}_{2}$ column chromatography (70-230 mesh; $320 \mathrm{~g})$ using $\mathrm{CHCl}_{3}$ - $\mathrm{EtOH}(10: 1 \rightarrow 5: 1)$ and $\mathrm{CHCl}_{3}-\mathrm{MeOH}(3: 1)$ as eluent to give eight fractions (CTB1-CTB8). The second fraction $\left(\mathrm{CTB} 2 ; 2.0 \mathrm{~g}\right.$ ) was subjected to further $\mathrm{SiO}_{2}$ column chromatography $(100 \mathrm{~g})$ and was eluted with $\mathrm{CHCl}_{3}-\mathrm{MeOH}$ (10:1) to provide pure DHC $(1.4 \mathrm{~g})$. The structure of DHC was analyzed by nuclear magnetic resonance (NMR; Varian Unity Inova AS-400 FT-NMR spectrometer; Varian Medical Systems, Inc., Palo Alto, CA, USA) and mass spectrometry (MS; JEOL JMSAX-700: JEOL Ltd., Tokyo, Japan).

Cell culture and expression vectors. The $\mathrm{C} 2 \mathrm{C} 12$ myoblast cells, 10T1/2 embryonic fibroblast cells and embryonic kidney $293 \mathrm{~T}$ cells were cultured as described previously (14). To induce differentiation of $\mathrm{C} 2 \mathrm{C} 12$ myoblasts, cells at near confluence were removed from growth medium (GM), which consisted of Dulbecco's modified Eagle's medium (DMEM) containing 15\% fetal bovine serum (FBS; Hyclone GE
Healthcare Life Sciences, Logan, UT, USA), and were cultured in differentiation medium (DM), which consisted of DMEM containing $2 \%$ horse serum (HS; Hyclone; GE Healthcare Life Sciences). Myotube formation was observed after 2 or 3 days of differentiation. The efficiency of myotube formation was quantified using a transient differentiation assay, as previously described (14). To generate $\mathrm{C} 2 \mathrm{C} 12$ cells that transiently overexpressed short hairpin RNAs (shRNAs) against Cdo, the cells were transfected with the indicated expression vector using Lipofectamine ${ }^{\circledR} 2000$ (Invitrogen; Thermo Fisher Scientific, Inc.) with $10 \mathrm{~T} 1 / 2$ and $293 \mathrm{~T}$ cells seeded at $3 \times 10^{5} / 10 \mathrm{~cm}$ cell culture plate containing DMEM, 10\% FBS and antibiotics, and the following day the cells were transfected with $10 \mu \mathrm{g}$ of MyoD construct or pcDNA vector as a control for 2 days at $37^{\circ} \mathrm{C}$. To produce $\mathrm{C} 2 \mathrm{C} 12$ cell lines that stably overexpress shRNA against $\mathrm{Cdo}, \mathrm{C} 2 \mathrm{C} 12$ cells were seeded at $1 \times 10^{5} / 10 \mathrm{~cm}$ cell culture plate containing DMEM, 15\% FBS and antibiotics. The cells were transfected for 1 day at $37^{\circ} \mathrm{C}$ with $10 \mu \mathrm{g}$ Cdo construct and pSuper vectors served as a control. Cultures were subsequently selected in puromycin-containing medium. Puromycin-resistant cells were pooled and analyzed. Cdo shRNA was purchased from Sigma-Aldrich (Merck Millipore, Darmstadt, Germany) and its sequence is 5'-CAGCGTTGG TGCCGTTGTG-3'. pSuper was obtained from Oligoengine (Seattle, WA, USA).

Western blot analysis and immunoprecipitation. Western blot analysis was performed as described previously (15). In C2C12 myoblasts, $\mathrm{C} 2 \mathrm{C} 12$ cells were treated with indicated concentrations of DHC and differentiation was induced for 2 or 3 days at $37^{\circ} \mathrm{C} .10 \mathrm{~T} 1 / 2$ and $293 \mathrm{~T}$ cells were treated with $500 \mathrm{nM}$ DHC. Following incubation for 1 day at $37^{\circ} \mathrm{C}$, cell lysates were subjected to immunoprecipitation with $\mathrm{E} 2 \mathrm{~A}$ antibodies followed by immunoblotting with MyoD antibodies. Briefly, cells were lysed in cell extraction buffer $[10 \mathrm{mM}$ Tris- $\mathrm{HCl}$ (pH 8.0), $150 \mathrm{mM} \mathrm{NaCl}, 1 \mathrm{mM}$ EDTA, $1 \%$ Triton X-100] containing a complete protease inhibitor cocktail (Roche Diagnostics, Indianapolis, IN, USA), and 30-50 $\mu \mathrm{g}$ proteins were separated by 6-10\% (6\% for MHC, Cdo and pan-cadherin antibodies, and $10 \%$ for all other antibodies) sodium dodecyl sulfate-polyacrylamide gel electrophoresis. The primary antibodies used were: Anti-MyoD (cat. no. sc-32758; mouse; 1:1,000), anti-myogenin (cat. no. sc-576; rabbit; 1:1,000), anti-p38 MAPK (cat. no. sc-728; anti-rabbit; 1:1,000), anti-E2A (cat. no. sc-763; rabbit; 1:1,00) (all Santa Cruz Biotechnology, Inc., Dallas, TX, USA), anti-phosphorylated (p)-p38 MAPK (p-p38; cat. no. 9211; rabbit; 1:500, Cell Signaling Technology, Inc., Beverly, MA, USA), anti-pan-Cadherin (cat. no. C3678; rabbit; Sigma-Aldrich; Merck Millipore), anti-Cdo (cat. no. AF2429; mouse; R\&D Systems, Minneapolis, MN, USA) and anti-MHC (cat. no. MF20; mouse; Developmental Studies Hybridoma Bank, Iowa City, IA, USA). Horseradish peroxidase-conjugated secondary antibodies from Santa Cruz Biotechnology, Inc. were diluted between 1:5,000 and 1:10,000 and used as follows: Goat anti-mouse IgG-HRP (cat. no. sc-2005); goat anti-rabbit IgG-HRP (cat. no. sc-2004); and donkey anti-goat IgG-HRP (cat. no. sc-2033). Primary antibodies were incubated overnight at $4{ }^{\circ} \mathrm{C}$ and secondary antibodies were incubated for $2-3 \mathrm{~h}$ at room temperature. For the immunoprecipitation assay, 293T ( $3 \times 10^{5} /$ plate) or 10T1/2 
cells $\left(3 \times 10^{5} /\right.$ plate) were transfected with $10 \mu \mathrm{g}$ MyoD (based on the pcDNA vector, accession number: NM-010866) using Lipofectamine $2000{ }^{\circledR}$ according to the manufacturer's protocol for $20 \mathrm{~min}$ at room temperature and were treated with DHC. After $24 \mathrm{~h}$ of treatment of $\mathrm{C} 2 \mathrm{C} 12,10 \mathrm{~T} 1 / 2$ and 293T cells with $500 \mathrm{~nm} \mathrm{DHC}$ at $37^{\circ} \mathrm{C}$, whole cell extracts were incubated with anti-E2A and protein $\mathrm{G}$ agarose beads (Roche Diagnostics) overnight at $4^{\circ} \mathrm{C}$. Subsequently, the beads were washed three times with extraction buffer, were resuspended in extraction buffer, and the samples were analyzed by western blotting.

Screening process. Phytochemicals purified from Corydalis tuber were screened using MyoD luciferase assay and MHC western blotting. For the MyoD luciferase assay, C2C12 cells were plated for $24 \mathrm{~h}$ in 24 -well plates and transiently transfected with the $4 R T K$ reporter gene using Lipofectamine 2000 (Invitrogen; Thermo Fisher Scientific, Inc.) according to the manufacturer's protocol. Following incubation for $24 \mathrm{~h}$, cells were treated with phytochemicals $(10 \mathrm{nM})$ from Corydalis tuber for an additional $24 \mathrm{~h}$. Luciferase activities were measured on a Berthold luminometer, integrating light emission over $20 \mathrm{sec}$. Transfection efficiencies were normalized by co-transfecting $50 \mathrm{ng}$ of the $\beta$-galactosidase plasmid, pCH110 (GE Healthcare Life Sciences). All transfections were performed in duplicate a minimum of three times. The second screening was conducted using MHC western blotting. $\mathrm{C} 2 \mathrm{C} 12$ cells were plated for $24 \mathrm{~h}$ in 6 -well plates, and treated with phytochemicals $(10 \mathrm{nM})$ from Corydalis tuber in $2 \%$ horse serum for an additional $48 \mathrm{~h}$. The cells were lysed and western blotting was performed. The primary antibody used was anti-MHC, and quantification of the signal was performed using Image Gauge software version 4.0 (Fujifilm, Tokyo, Japan).

Immunocytochemistry and confocal microscopy. Immunostaining for MHC expression was performed as described previously (14). Briefly, C2C12 cells were treated with 125,250 and $500 \mathrm{nM}$ DHC for 3 days at $37^{\circ} \mathrm{C}$, fixed with $4 \%$ paraformaldehyde, permeabilized with $0.1 \%$ Triton $\mathrm{X}-100$ in phosphate-buffered saline (PBS), and were blocked. Subsequently, the cells were stained with anti-MHC, followed by incubation with an Alexa Fluor 594-conjugated secondary antibody (cat. no. A11012; 1:500; Molecular Probes; Thermo Fisher Scientific, Inc.). Primary antibodies were incubated overnight at $4^{\circ} \mathrm{C}$ and secondary antibodies were incubated for $2 \mathrm{~h}$ at room temperature. Images were captured and processed using a Nikon ECLIPSE TE-2000 U microscope and NIS-Elements F software (Nikon Corporation, Tokyo, Japan). The quantitative differentiation assay was performed at least three times. To observe the effects of DHC on p38 phosphorylation in $\mathrm{C} 2 \mathrm{C} 12$ cells, $\mathrm{C} 2 \mathrm{C} 12$ cells were treated with $500 \mathrm{nM}$ DHC for 2 or 3 days at $37^{\circ} \mathrm{C}$ and were fixed with $4 \%$ paraformaldehyde. Cultures were subsequently permeabilized with $0.1 \%$ Triton $\mathrm{X}-100$ in PBS, blocked in $5 \%$ horse serum in $0.1 \%$ Triton $\mathrm{X}-100$ in PBS, and were incubated with anti-p-p38 followed by an incubation with an Alexa Fluor 594-conjugated secondary antibody. Nuclei were counterstained with 4',6-diamidino-2-phenylindole (DAPI; cat. no. sc-3598; Santa Cruz Biotechnology, Inc.). Images were obtained using a Zeiss LSM-510 Meta confocal microscope
(Carl Zeiss AG, Oberkochen, Germany). Quantification of the p-p38 fluorescent signal was performed using Image Gauge software version 4.0.

Bromodeoxyuridine (BrdU) assay. C2C12 cells were cultured in 6-well plates and were treated with dimethyl sulfoxide (DMSO) or $500 \mathrm{nM} \mathrm{DHC}$ in GM for $24 \mathrm{~h}$ at $37^{\circ} \mathrm{C}$. After $24 \mathrm{~h}$ of treatment, $10 \mu \mathrm{M}$ BrdU (cat. no. 550891; $10 \mu \mathrm{g} / \mathrm{ml}$; BD Biosciences, San Jose, CA, USA) was added to the DMSOor DHC-treated cells and was incubated for $30 \mathrm{~min}$ at $37^{\circ} \mathrm{C}$. The cells were fixed and immunostained with anti-BrdU (cat. no. sc-32323; mouse; 1:500; Santa Cruz Biotechnology, Inc.) overnight at $4^{\circ} \mathrm{C}$, followed by incubation for $2 \mathrm{~h}$ at room temperature with a fluorescein isothiocyanate-conjugated secondary antibody (cat. no. A11001; 1:500; Invitrogen; Thermo Fisher Scientific, Inc.). Images were captured and processed using a Nikon ECLIPSE TE-2000 U microscope and NIS-Elements F software. The quantitative differentiation assay was performed at least three times.

Statistical analysis. Data are presented as the mean \pm standard deviation from at least three independent experiments. Data were analyzed using the Student's t-test. For comparisons between multiple groups, statistical significance was determined using one-way analysis of variance and the Scheffe test for post-hoc analysis. Statistical analysis was conducted using SPSS 18.0 (SPSS, Inc., Chicago, IL, USA). P<0.05 was considered to indicate a statistically significant difference.

\section{Results and Discussion}

DHC enhances myogenic differentiation. To identify effective activators of myoblast differentiation, phytochemicals purified from Corydalis tuber were screened, a luciferase assay was performed to measure MyoD activity, and western blotting was conducted to detect the expression levels of MHC. An active component was identified from the alkaloidal fraction of Corydalis tuber (Fig. 1A). Its molecular weight was determined to be 366 from electron ionization-MS spectra, and 1,2,4,5-tetrasubstituted benzene signals (7.18 and $6.94 \mathrm{ppm}$ ), 1,2,3,4-tetrasubstituted benzene signals (7.96 and $7.91 \mathrm{ppm}$ ), four methoxy signals $(4.28,4.07,4.00$ and $3.95 \mathrm{ppm})$ and an allylmethyl proton signal (2.98 ppm) were observed in ${ }^{1} \mathrm{H}-\mathrm{NMR}$ spectrum. The same pattern of ${ }^{13} \mathrm{C}-\mathrm{NMR}$ spectroscopic data also revealed the typical spectroscopic pattern of the isoquinoline alkaloid. The structure of the active compound was finally confirmed to be DHC by comparing the data with those reported in the literature (16).

The present study initially aimed to confirm whether DHC was able to enhance myoblast differentiation. C2C12 cells were induced to differentiate for 2 days in the presence of varying concentrations of DHC (125-500 nM). DHC-treated C2C12 cells exhibited increased expression of muscle-specific proteins including MHC, MyoD and myogenin in a dose-dependent manner compared with in the control DMSO-treated cells (Fig. 1B). However, the expression levels of the promyogenic receptor Cdo were not markedly altered. To determine whether treatment with DHC enhanced the formation of myotubes, DMSO- and DHC-treated C2C12 cells were induced to differentiate for 3 days, and were fixed and 
immunostained with anti-MHC followed by DAPI staining. As shown in Fig. 1C, DHC-treated C2C12 cells formed larger myotubes with more nuclei per myotube compared with the control cells. The MHC-positive cells were scored as follows: Mononucleated, containing 2-5 nuclei, containing 6-9 nuclei, or containing $\geq 10$ nuclei (Fig. 1D). In concordance with Fig. 1C, C2C12 cells treated with DHC exhibited a significant increase in myotubes with $\geq 10$ nuclei, relative to the vehicle-treated cells (Fig. 1D). Consistently, treatment with DHC increased the protein expression levels of $\mathrm{MHC}, \mathrm{MyoD}$ and myogenin (Fig. 1E). In addition, DHC enhanced the activity of ectopically expressed MyoD in non-muscle cells, including 10T1/2 fibroblasts and 293T cells (data not shown). To determine whether DHC affects myoblast proliferation, a BrdU incorporation assay was conducted to assess proliferation. As shown in Fig. 1F and G, treatment with DHC had no obvious effect on the proliferative capacities of $\mathrm{C} 2 \mathrm{C} 12$ myoblasts compared with the control-treated cells. These data suggest that DHC treatment promotes myoblast differentiation at the morphological and biochemical level.

DHC activates p38 MAPK in myoblast differentiation. Several signaling pathways activate p38 MAPK, which in turn induces transcriptional activation of MyoD and promotes myoblast differentiation $(14,15,17,18)$. To investigate whether DHC activates MyoD via p38 MAPK, C2C12 cells were induced to differentiate in the presence of DMSO or three concentrations of DHC, and p38 MAPK activation was analyzed. Treatment of $\mathrm{C} 2 \mathrm{C} 12$ cells with $\mathrm{DHC}$ upregulated the expression levels of active p-p38 in a dose-dependent manner (Fig. 2A and B). In addition, DHC-treated C2C12 cells exhibited strongly elevated levels of p-p38 at day 2, compared with the vehicle-treated cells (Fig. 2C and D).

The present study aimed to confirm whether DHC treatment enhanced phosphorylation of p38 by immunostaining and confocal microscopy. $\mathrm{C} 2 \mathrm{C} 12$ cells were induced to differentiate for 1 day, fixed and immunostained with antibodies against p-p38, followed by DAPI staining to visualize nuclei. As presented in Fig. 2E, DHC-treated C2C12 cells displayed an increase in cells positive for p-p38 signals compared with the control cells. Subsequently, p-p38 signal intensity was quantified relative to that of DMSO-treated cells, which was set to 1.0. DHC-treated C2C12 cells displayed a 2.34-fold increase in p-p38 signal intensity (Fig. 2F). Previous studies have demonstrated that p38 MAPK is a key regulator for MyoD activation to promote myoblast differentiation (19). These results indicate that DHC promotes myoblast differentiation via activation of $\mathrm{p} 38 \mathrm{MAPK}$ and MyoD.

DHC enhances the heterodimerization of MyoD with E proteins. The transcriptional activity of MyoD is regulated through several regulatory pathways, including heterodimerization of MyoD with E proteins, which is stimulated by p38 MAPK-mediated phosphorylation of E proteins $(4,20)$. Since DHC treatment activated p38 MAPK and MyoD, the present study investigated whether DHC regulates MyoD activity through enhancing interactions between MyoD and an $\mathrm{E}$ protein $(\mathrm{E} 2 \mathrm{~A})$ in myoblast differentiation. $\mathrm{C} 2 \mathrm{C} 12$ cells were treated with DHC, differentiated, and were harvested at D2. Cell lysates were then subjected to immunoprecipitation with an E2A antibody followed by immunoblotting with MyoD and E2A antibodies. DHC-treated C2C12 cells exhibited markedly increased precipitation of MyoD proteins with E2A compared with the control cells (Fig. 3A). In addition, total E2A levels appeared to be slightly increased by DHC treatment. Control immunoprecipitation with immunoglobulin $\mathrm{G}$ did not precipitate MyoD (data not shown). These results were further confirmed using 10T1/2 embryonic fibroblasts transiently transfected with MyoD expression vectors. Cells were treated with DMSO or DHC and incubated for $24 \mathrm{~h}$, followed by immunoprecipitation analysis. As shown in Fig. 3B, DHC treatment enhanced the interaction of MyoD with E2A in 10T1/2 mouse embryonic fibroblasts. In the lysates, the protein levels of E2A remained constant, whereas the protein levels of MyoD were increased in DHC-treated cells. In agreement with these results, DHC treatment also increased the binding of MyoD to E2A proteins in MyoD-transfected 293 T cells (Fig. 3C). Activation of MyoD in paired box (Pax)3/Pax7/MyoD-expressing myoblasts has been shown to sequentially induce alterations in muscle-specific gene expression, thus leading to terminal differentiation of myoblasts into multinucleated myotubes and myofibers (4). MyoD binds to the MyoD-responsive element E-box in the promoter regions of downstream muscle target genes, thereby inducing the transcription of these muscle-related genes in collaboration with myocyte enhancer factor-2 (MEF2) (21). In the transcriptional regulation of MyoD, p38 MAPK has an important role in heterodimerization of MyoD with E proteins (7). Notably, it has been reported that tetrahydropalmatine, which is an alkaloid compound purified from medicinal herbs, promotes transdifferentiation of 10T1/2 fibroblasts into myoblasts mediated by MyoD (11). Therefore, natural compounds, including DHC, may elevate the transcriptional activity of MyoD in non-muscle cells, and this property may be beneficial in transdifferentiation of non-muscle cells into myoblasts. These data suggest that DHC upregulates the interaction of MyoD with E2A via activation of p38 MAPK.

DHC rescues myogenic differentiation in Cdo-depleted C2C12 cells. The promyogenic receptor Cdo has been reported to act as a critical component that integrates cell contact-mediated signals from the cell surface into the myogenic regulatory network via 338 MAPK and MyoD activation (8). In a previous study, Cdo depletion or deficiency in myoblasts resulted in reduced p38 MAPK and MyoD activation, which in turn induced defective myoblast differentiation (15). The results of the present study suggested that DHC may increase myoblast differentiation via activation of p38 MAPK. Therefore, experiments were conducted to determine whether defective myoblast differentiation induced by Cdo depletion can be rescued by DHC treatment. C2C12 cells were transfected with pSuper and Cdo shRNA expression vectors. C2C12/pSuper and C2C12/Cdo shRNA cells were subsequently treated with DMSO or DHC, and were induced to differentiate for 2 days, followed by western blot analysis. In agreement with our previous study (11), Cdo-depleted cells (Fig. 4A) exhibited decreased levels of MHC, MyoD and p-p38 compared with in the C2C12/pSuper control cells (Fig. 4B). Treatment of C2C12/Cdo shRNA cells with $\mathrm{DHC}$ restored the expression of muscle-specific proteins 
A

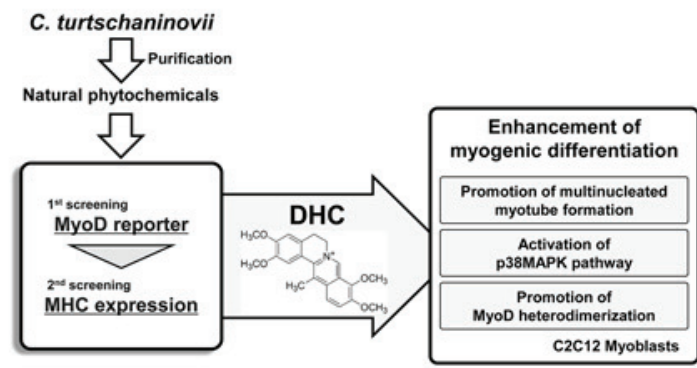

B

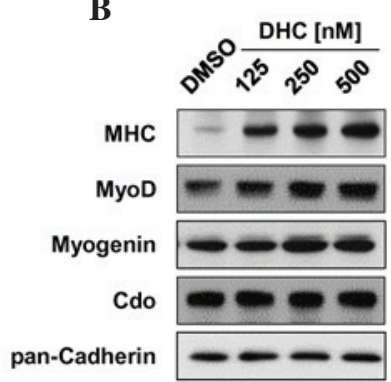

C
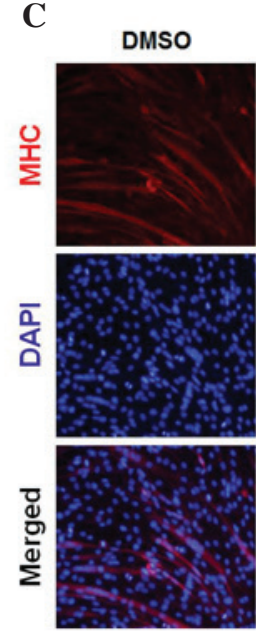

E

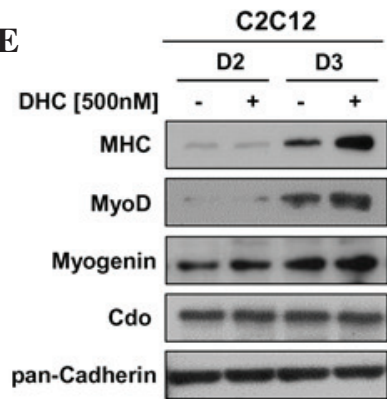

DHC [nM]
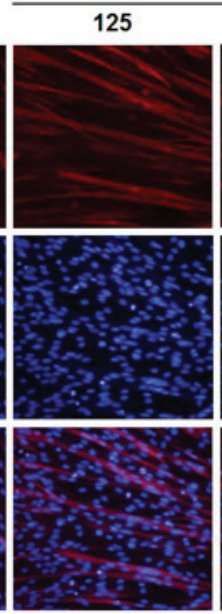

F

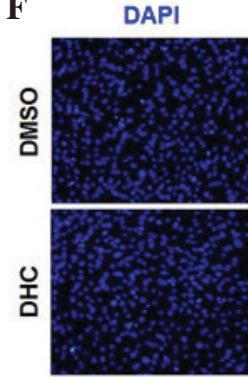

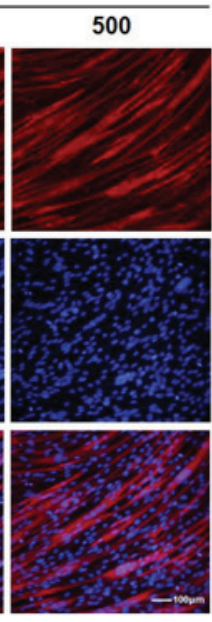

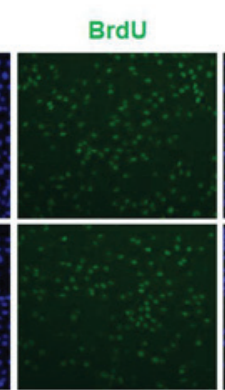

D

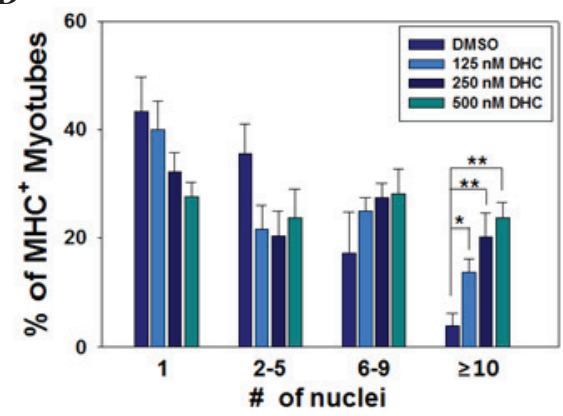

Figure 1. Dehydrocorydaline (DHC) enhances myogenic differentiation. (A) Schematic diagram indicating the experimental procedure for screening of natural compounds. (B) $\mathrm{C} 2 \mathrm{C} 12$ cells were treated with the indicated amounts of DHC or dimethyl sulfoxide (DMSO) and were induced to differentiate for 2 days (D2). Lysates underwent western blotting with antibodies against myosin heavy chain (MHC), MyoD, myogenin, Cdo, and pan-Cadherin as a loading control. (C) $\mathrm{C} 2 \mathrm{C} 12$ cells were treated with DMSO or various concentrations of DHC and were induced to differentiate for 3 days (D3). The cells were immunostained with MHC antibodies followed by 4',6-diamidino-2-phenylindole (DAPI) staining to visualize nuclei. Scale bar, $100 \mu \mathrm{m}$. (D) Quantification of myotube formation in the cells presented in (C). Data are presented as the mean \pm standard deviation (SD) of three determinations. The experiment was repeated three times with similar results. ${ }^{*}<0.05$ and ${ }^{* *} \mathrm{P}<0.01$ vs. the DMSO-treated group. (E) $\mathrm{C} 2 \mathrm{C} 12$ cells were treated with $500 \mathrm{nM}$ DHC for D2 and D3. Lysates were immunoblotted with antibodies against MHC, MyoD, myogenin, Cdo, and pan-Cadherin as a loading control. (F) C2C12 cells were treated with DMSO or DHC for 1 day and were labeled with bromodeoxyuridine (BrdU) for 30 min followed by immunostaining with anti-BrdU and DAPI staining to visualize nuclei. Scale bar, $100 \mu \mathrm{m}$. (G) Quantification of BrdU-positive cells presented in (F). Data are presented as the mean \pm SD of three determinations. The experiment was repeated three times with similar results. n.s., not significant.

to similar levels observed in DHC-treated C2C12/pSuper cells (Fig. 4B). In addition, DHC-treated C2C12/Cdo shRNA cells exhibited slightly higher levels of p-p38 compared with in the DHC-treated C2C12/pSuper cells, whereas total p38 protein expression remained constant.

The present study also investigated whether defective myotube formation in Cdo-depleted cells can be improved by DHC treatment. C2C12/pSuper and C2C12/Cdo shRNA cells were treated with DMSO or DHC, and were induced to differentiate for 3 days, followed by immunostaining analysis for myotube formation. $\mathrm{C} 2 \mathrm{C} 12 / \mathrm{pSuper}$ cells treated with DHC exhibited enhanced myotube formation, and myotubes contained $\geq 10$ nuclei, as compared with the control-treated C2C12/pSuper cells. Vehicle-treated C2C12/Cdo shRNA cells exhibited reduced myotube formation, which was markedly improved following DHC treatment, almost to control cell levels (Fig. 4C and D). Cdo is a promyogenic receptor protein, which regulates myoblast differentiation predominantly via p38 MAPK activation (18). During myoblast differentiation, Cdo forms multiprotein complexes to activate the p38 MAPK pathway via direct binding of the Cdo cytoplasmic tail to scaffold proteins c-Jun NH2-terminal kinase-associated leucine zipper protein and BCL2/adenovirus E1B $19 \mathrm{kDa}$-interacting protein 2 (17,18). p38 MAPK 
A

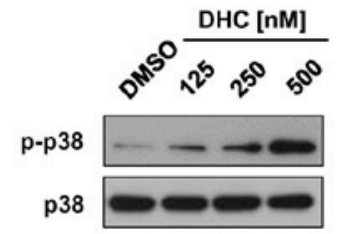

C

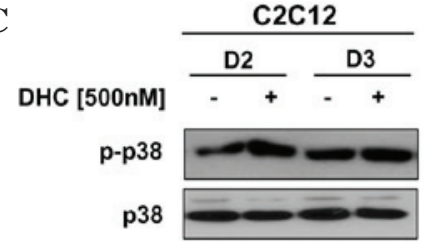

B

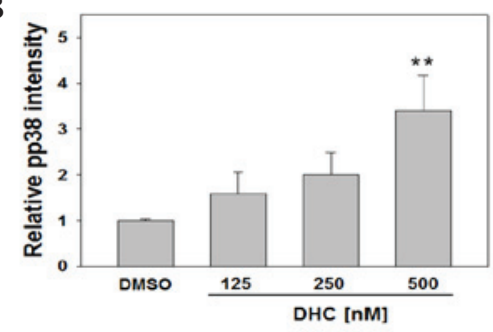

D

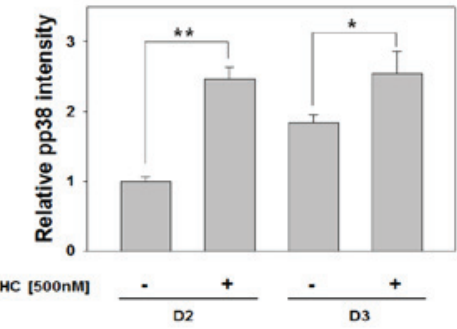

E
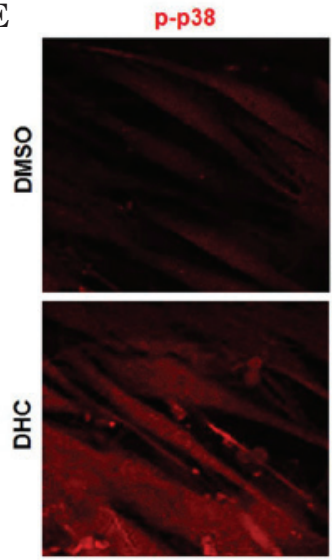

DAPI
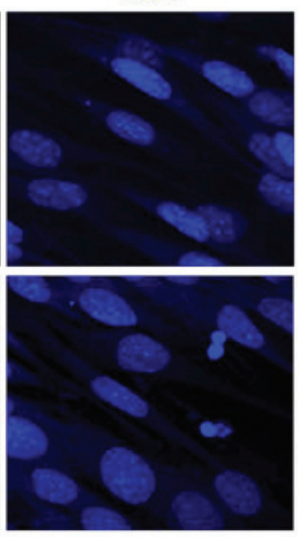

Merged

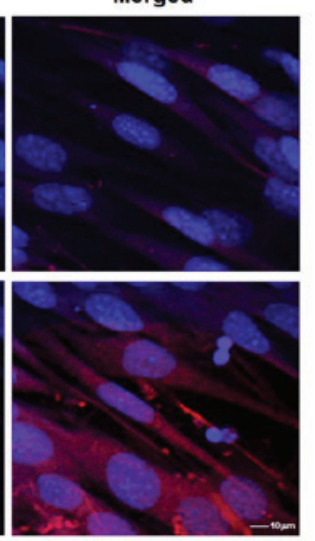

F

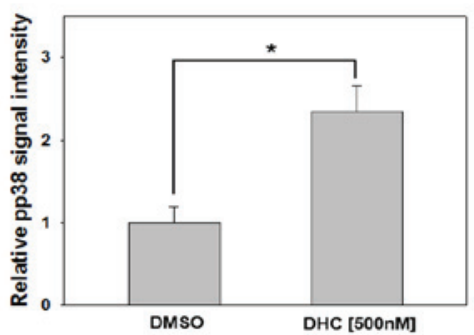

Figure 2. Dehydrocorydaline (DHC) activates p38 mitogen-activated protein kinases (MAPK) in myoblast differentiation. (A) C2C12 cells were treated with DHC and induced to differentiate for 2 days (D2). Lysates underwent western blotting with antibodies against phosphorylated-p38 MAPK (p-p38) and total p38. (B) Quantification of three replicate immunoblots, one of which is presented in (A). The intensity of the p-p38 and p38 signals were quantified, and the values were obtained from a ratio of p-p38/p38. Data are presented as the mean \pm standard deviation (SD) from triplicate determinations. ${ }^{*} \mathrm{P}<0.05$ and ${ }^{* *} \mathrm{P}<0.01$ vs. the dimethyl sulfoxide (DMSO)-treated group. (C) $\mathrm{C} 2 \mathrm{C} 12$ cells were treated with DHC for D2 and 3 days (D3). Lysates were immunoblotted with antibodies against p-p38 and total p38. (D) Quantification of three replicate immunoblots, one of which is presented in (C). The intensity of the p-p38 and p38 signals were quantified, and the values were obtained from a ratio of $\mathrm{p}-\mathrm{p} 38 / \mathrm{p} 38$. Data are presented as the mean $\pm \mathrm{SD}$ from triplicate determinations. $\mathrm{P}<0.05$ and ${ }^{* *} \mathrm{P}<0.01$ vs. the DMSO-treated group. (E) $\mathrm{C} 2 \mathrm{C} 12$ cells were treated with DMSO or DHC, fixed and stained with antibodies against p-p38 (red), followed by 4',6-diamidino-2-phenylindole (DAPI) staining (blue). Scale bar, $10 \mu \mathrm{m}$. (F) Quantification of p-p38-positive cells presented in (D). Data are presented as the mean \pm SD from triplicate determinations. The experiment was repeated three times with similar results. ${ }^{*} \mathrm{P}<0.05$.

A

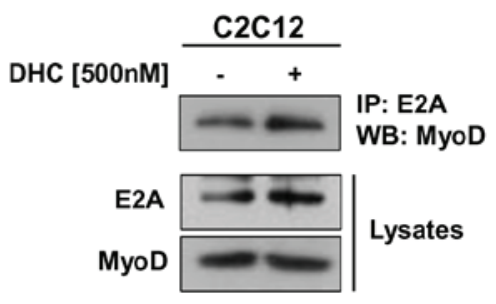

B

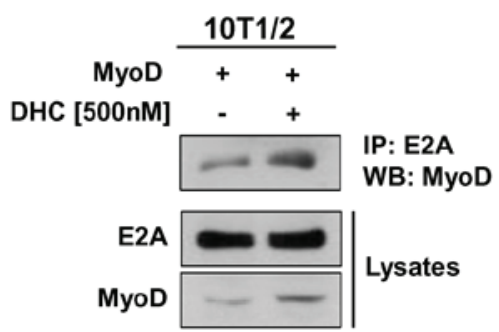

C

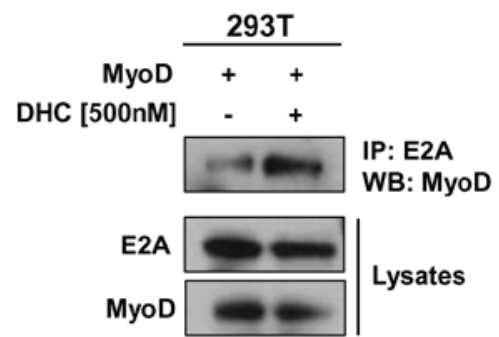

Figure 3. Treatment with dehydrocorydaline (DHC) enhances heterodimerization of MyoD with E2A. (A) C2C12, (B) MyoD-transfected 10T1/2 and (C) MyoD-transfected 293T cells were treated with dimethyl sulfoxide (DMSO) or DHC. Cell lysates were subjected to immunoprecipitation with E2A antibodies followed by immunoblotting with MyoD antibodies. Total lysates served as expression controls. The experiment was repeated three times with similar results.

subsequently regulates the activation of key muscle transcription factors, including MyoD and MEF2C (22). The results of the present study demonstrated that DHC ameliorated Cdo depletion-induced defective differentiation via p38 MAPK 
A

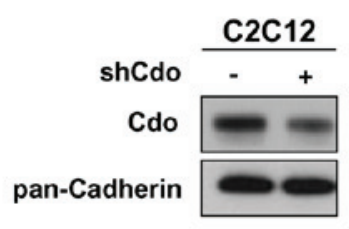

$\mathrm{C}$

$\mathbf{D}$
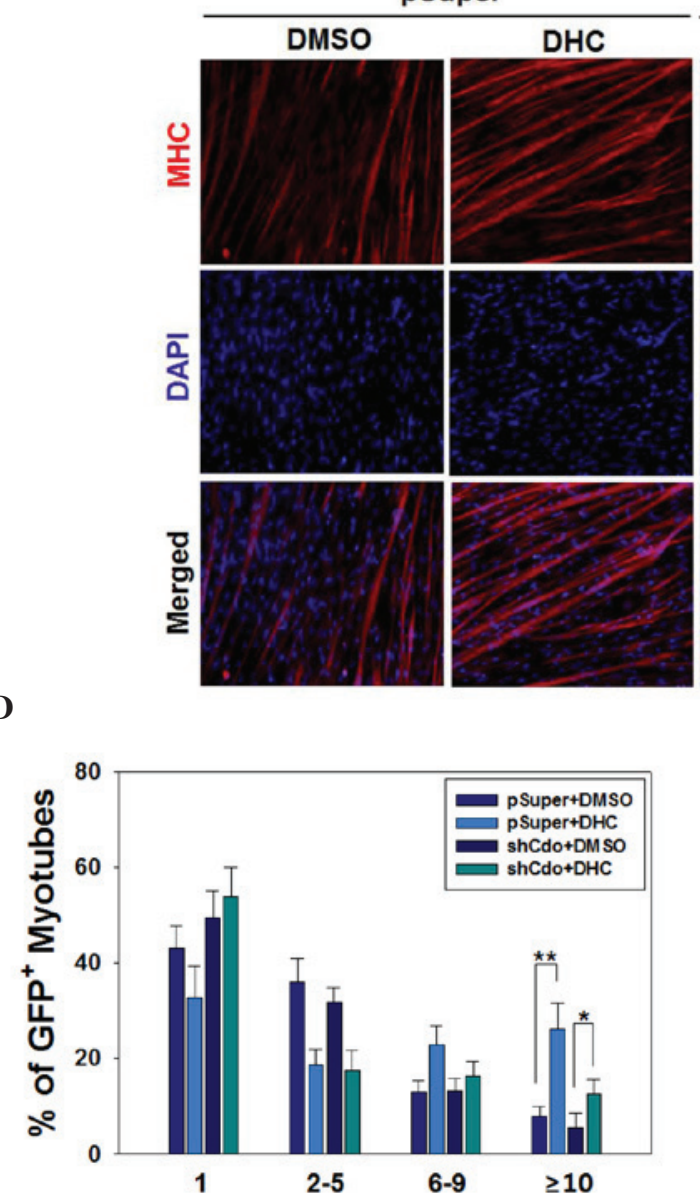

B

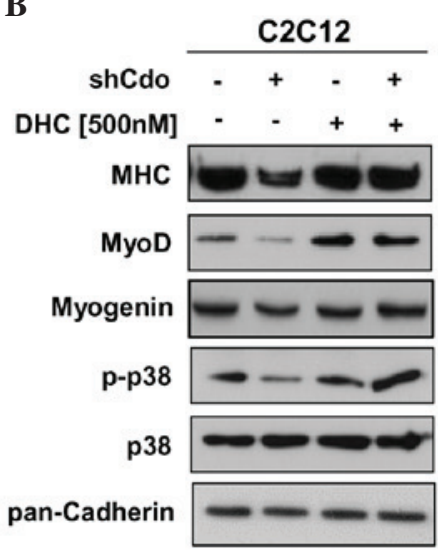

shCdo
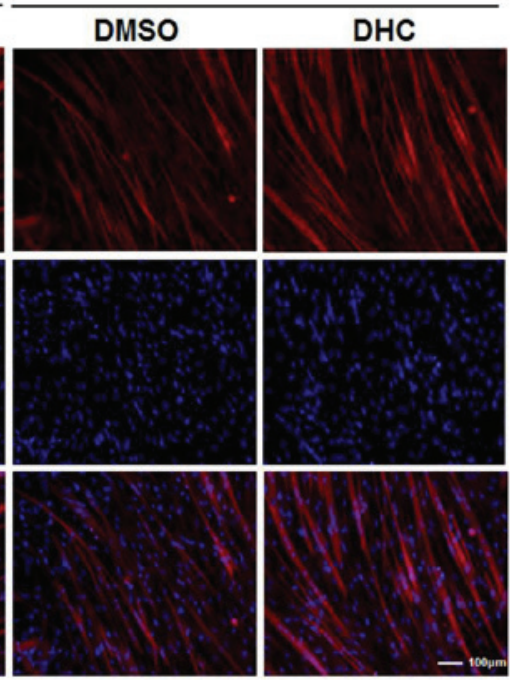

$\mathbf{E}$

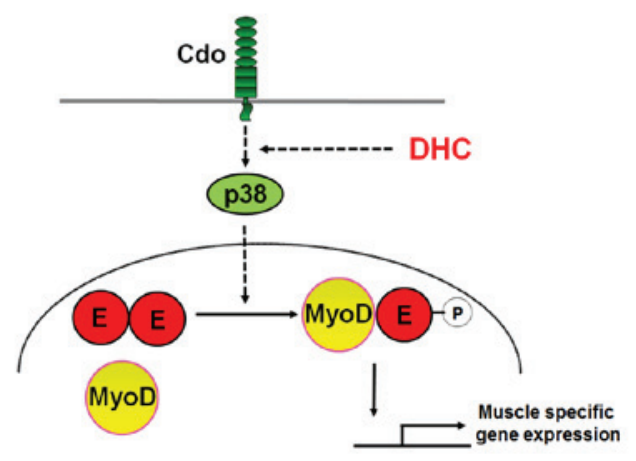

Figure 4. Dehydrocorydaline (DHC) rescues myogenic differentiation in Cdo-depleted C2C12 cells. (A) C2C12 cells were transfected with control or Cdo short hairpin (sh)RNA vectors, and lysates were analyzed for Cdo expression. Pan-Cadherin was used as a loading control. (B) C2C12/pSuper and C2C12/Cdo shRNA cells were treated with dimethyl sulfoxide (DMSO) or DHC. The lysates were subjected to western blotting with antibodies against myosin heavy chain (MHC), MyoD, myogenin, phosphorylated-p38 mitoren-activated protein kinases (MAPK) (p-p38) and p38. (C) C2C12/pSuper and C2C12/Cdo shRNA cells were fixed and stained with antibodies against MHC, followed by 4',6-diamidino-2-phenylindole (DAPI) staining to visualize cell nuclei. Scale bar, $10 \mu \mathrm{m}$. (D) Quantification of myotube formation as presented in (C). Data are presented as the mean \pm standard deviation of triplicate determinations. The experiment was repeated three times with similar results. ${ }^{*} \mathrm{P}<0.05$ and ${ }^{* * *} \mathrm{P}<0.01$ vs. the DMSO-treated cells. (E) Model of DHC-stimulated Cdo/p38 MAPK signaling during myogenic differentiation. p3 8MAPK phosphorylates E proteins (E), leading to enhancement of heterodimerization with the myogenic basic helix-loop-helix factor, MyoD. This heterodimer can induce the expression of genes required for myogenic differentiation.

activation, which is consistent with the results of our previous study (18). Our previous study demonstrated that reactivation of p38 MAPK via constitutively activated MKK6 (MKK6EE), which is an upstream kinase of p38 MAPK, restored the differentiation capacity of Cdo-deficient myoblasts.
In conclusion, DHC isolated from Corydalis tuber significantly promoted myoblast differentiation via the activation of p38 MAPK and MyoD. DHC enhanced the expression of muscle-specific proteins, including $\mathrm{MHC}$, MyoD and myogenin. Activation of the p38 MAPK pathway 
by DHC increased the heterodimerization of MyoD and E proteins, resulting in MyoD activation (Fig. 4E). These findings provide evidence for the promyogenic effects of $\mathrm{DHC}$, and a mechanistic rationale for the potential pharmaceutical application of DHC in improvement of muscle regeneration to treat muscle atrophy.

\section{Acknowledgements}

The present study was supported by the Basic Science Research Program through the National Research Foundation of Korea (NRF) funded by the Ministry of Education, Science and Technology (grant no. 2013R1A1A2010280), an NRF grant funded by the Ministry of Science, ICT and Future Planning (grant no.2015R1A2A1A15056117), and an NRF grant funded by the Korea Government (MSIP) (grant no. NRF-2011-0030074).

\section{References}

1. Tedesco FS, Dellavalle A, Diaz-Manera J, Messina G and Cossu G: Repairing skeletal muscle: Regenerative potential of skeletal muscle stem cells. J Clin Invest 120: 11-19, 2010.

2. Mangner N, Adams V, Sandri M, Hoellriegel R, Hambrecht R, Schuler G and Gielen S: Muscle function and running activity in mouse models of hereditary muscle dystrophy: Impact of double knockout for dystrophin and the transcription factor MyoD. Muscle Nerve 45: 544-551, 2012.

3. Horsley V and Pavlath GK: Forming a multinucleated cell: Molecules that regulate myoblast fusion. Cells Tissues Organs 176: 67-78, 2004.

4. Berkes CA and Tapscott SJ: MyoD and the transcriptional control of myogenesis. Semin Cell Dev Biol 16: 585-595, 2005.

5. Lluís F, Perdiguero E, Nebreda AR and Muñoz-Cánoves P: Regulation of skeletal muscle gene expression by p38 MAP kinases. Trends Cell Biol 16: 36-44, 2006.

6. Davis RL, Cheng PF, Lassar AB and Weintraub H: The MyoD DNA binding domain contains a recognition code for muscle-specific gene activation. Cell 60: 733-746, 1990.

7. Lluís F, Ballestar E, Suelves M, Esteller M and Muñoz-Cánoves P: E47 phosphorylation by p38 MAPK promotes MyoD/E47 association and muscle-specific gene transcription. EMBO J 24: 974-984, 2005

8. Krauss RS, Cole F, Gaio U, Takaesu G, Zhang W and Kang JS: Close encounters: Regulation of vertebrate skeletal myogenesis by cell-cell contact. J Cell Sci 118: 2355-2362, 2005.

9. Kubo M, Matsuda H, Tokuoka K, Ma S and Shiomoto H: Anti-inflammatory activities of methanolic extract and alkaloidal components from Corydalis tuber. Biol Pharm Bull 17: 262-265, 1994.
10. Yun KJ, Shin JS, Choi JH, Back NI, Chung HG and Lee KT: Quaternary alkaloid, pseudocoptisine isolated from tubers of Corydalis turtschaninovi inhibits LPS-induced nitric oxide, PGE(2) and pro-inflammatory cytokines production via the down-regulation of NF-kappaB in RAW 264.7 murine macrophage cells. Int Immunopharmacol 9: 1323-1331, 2009.

11. Lee SJ, Yoo M, Go GY, Hwang J, Lee HG, Kim YK, Seo DW, Baek NI, Ryu JH, Kang JS and Bae GU: Tetrahydropalmatine promotes myoblast differentiation through activation of p38MAPK and MyoD. Biochem Biophys Res Commun 455: 147-152, 2014.

12. Ishiguro $\mathrm{K}$, Ando $\mathrm{T}$, Maeda $\mathrm{O}$, Watanabe $\mathrm{O}$ and Goto $\mathrm{H}$ : Dehydrocorydaline inhibits elevated mitochondrial membrane potential in lipopolysaccharide-stimulated macrophages. Int Immunopharmacol 11: 1362-1367, 2011.

13. Xu Z, Chen X, Fu S, Bao J, Dang Y, Huang M, Chen L and Wang Y: Dehydrocorydaline inhibits breast cancer cells proliferation by inducing apoptosis in MCF-7 cells. Am J Chin Med 40: 177-185, 2012.

14. Bae GU, Kim BG, Lee HJ, Oh JE, Lee SJ, Zhang W, Krauss RS and Kang JS: Cdo binds Abl to promote p38alpha/beta mitogen-activated protein kinase activity and myogenic differentiation. Mol Cell Biol 29: 4130-4143, 2009.

15. Tran P, Ho SM, Kim BG, Vuong TA, Leem YE, Bae GU and Kang JS: TGF- $\beta$-activated kinase 1 (TAK1) and apoptosis signal-regulating kinase 1 (ASK1) interact with the promyogenic receptor Cdo to promote myogenic differentiation via activation of p38MAPK pathway. J Biol Chem 287: 11602-11615, 2012.

16. Slavík J and Slavíková L: Alkaloids from Corydalis cava (L). SCHW. et KOERTE. Collect Czech Chem Commun 44: 2261-2274,1979.

17. Kang JS, Bae GU, Yi MJ, Yang YJ, Oh JE, Takaesu G, Zhou YT, Low BC and Krauss RS: A Cdo-Bnip-2-Cdc42 signaling pathway regulates p38alpha/beta MAPK activity and myogenic differentiation. J Cell Biol 182: 497-507, 2008.

18. Takaesu G, Kang JS, Bae GU, Yi MJ, Lee CM, Reddy EP and Krauss RS: Activation of p38alpha/beta MAPK in myogenesis via binding of the scaffold protein JLP to the cell surface protein Cdo. J Cell Biol 175: 383-388, 2006.

19. Keren A, Tamir Y and Bengal E: The p38 MAPK signaling pathway: A major regulator of skeletal muscle development. Mol Cell Endocrinol 252: 224-230, 2006.

20. Davis RL, Weintraub H and Lassar AB: Expression of a single transfected cDNA converts fibroblasts to myoblasts. Cell 51: 987-1000, 1987.

21. Puri PL and Sartorelli V: Regulation of muscle regulatory factors by DNA-binding, interacting proteins, and post-transcriptional modifications. J Cell Physiol 185: 155-173, 2000.

22. Zetser A, Gredinger E and Bengal E: p38 mitogen-activated protein kinase pathway promotes skeletal muscle differentiation. Participation of the Mef2c transcription factor. J Biol Chem 274: 5193-5200, 1999. 